and 4) whether there is a remaining association not mediated by these factors.

Methods We used cross-sectional data from the Canadian Longitudinal Study on Aging $(\mathrm{N}=17,938)$, focusing on working, non-retired adults aged 45-64 years. Data were collected via telephone and in-person interview over the 2011-2015 period. Functional, psychosocial, organizational and life-stage factors were measured using existing variables to create composite index scores. Path models examined the relationship between age and retirement expectations, and the proportion of the relationship explained via each factor.

Results Age was associated with functional, psychosocial, organizational and life-stage scores in expected directions. Older age also was associated with earlier retirement expectations. Path models found that $25-30 \%$ of the total relationship between older age and retirement expectations was mediated through life-stage and organizational factors.

Conclusion Our study demonstrates the feasibility of measuring functional, psychosocial, organizational and life-stage concepts via existing data to better understand age-related inequalities in retirement expectations. Future research should focus on measuring additional items for psychosocial and organizational factors, followed by validation of the extent to which each item explains age differences in other work outcomes.

\section{0-439 THE COMPLEX ASSOCIATION OF PERCEIVED WORKPLACE SAFETY, WORK ENVIRONMENT, AND NATIONAL FACTORS WITH THE MENTAL HEALTH OF AGING WORKERS IN EUROPE DURING THE COVID-19 PANDEMIC}

Shuli Bramli-Grinberg, David Christiani, 'Lilah Rinsky Halivni. ${ }^{1}$ Hebrew University, Israel

\subsection{6/OEM-2021-EPI.4}

Introduction The widespread COVID-19 contagion in workplaces has created a new workplace hazard, albeit investigated mainly among health care workers. Work-environment factors are related to workplace infection risks and individual vulnerability factors, like older age, predispose workers to severe illness. The stress and anxiety associated with the concerns regarding workplace safety and COVID-19 repercussions are jeopardizing aging workers' mental health (MH).

Objectives We aimed at investigating the individual and macro-level factors associated with declines in the $\mathrm{MH}$ of aging workers from different industry sectors. We hypothesize that higher perceived workplace safety is crucial in protecting their $\mathrm{MH}$ and mediates the work-environment influences.

Methods Using the Health, Ageing and Retirement in Europe (SHARE) data from COVID-19 survey (summer 2020) from 27 countries in Europe and Israel and additional data collected from pre-pandemic waves, we performed multi-level and mediation analyses to characterize work-environment, safety perception, socio-demographic, clinical, and nationallevel factors associated with $\mathrm{MH}$ among workers aged 50-70. Results Multi-level analyses demonstrated that $24 \%$ of the aging workers experience $\mathrm{MH}$ declines characterized by EastWest geographical European gradient associated with disease burden. The perceived workplace safety, which is low among $10 \%$ of the workers, is the strongest predictor- explaining $30 \%$ of their $\mathrm{MH}$ status and mediates the effects of workenvironment aspects, such as workplace contagion risk. Being a woman, having financial difficulties, a higher vulnerability index (comorbidities and age $>60$ ), pre-existing mental morbidity, and the national high burden of COVID-19 are associated with declines in $\mathrm{MH}$, whereas exclusively working on-site is protective.

Conclusion Evaluating workplace conditions and screening vulnerable sub-groups among the aging workers who are more prone to $\mathrm{MH}$ declines are imperative. Workplace interventions, integrated with individual targeted approaches to reduce the influence of work-environment factors on infection risks and mental distress, hence elevating workplace safety perception, are recommended.

\section{0-447 ASSOCIATION OF PERCEIVED JOB SECURITY AND CHRONIC HEALTH CONDITIONS WITH RETIREMENT IN OLDER UK AND U.S WORKERS}

${ }^{1}$ Miriam Mutambudzi, Evangelia Demou. 'Syracuse University, United States

\subsection{6/OEM-2021-EPI.5}

Background The relationship between job insecurity, chronic health conditions (CHCs), and retirement among older workers are likely to differ between countries that have different labor markets and health and social safety nets. To date, there are no epidemiological studies that have prospectively assessed the role of job insecurity in retirement incidence, while accounting for $\mathrm{CHCs}$ in two countries with vastly different welfare systems. We investigated the strength of the association baseline job insecurity and retirement incidence over an 11-year period while accounting for CHCs, among workers aged 50 and above in the UK and U.S.

Methods We performed Cox proportional hazards regression analysis, using data from the Health and Retirement Study (HRS [U.S. cohort, $\mathrm{n}=491$ ]) and English Longitudinal Study on Aging (ELSA [UK cohort $n=821]$ ).

Results We found evidence of reduced likelihood of retirement among job insecure adults in both cohorts, and a significant association between CHCs and retirement in the U.S cohort only. In the UK cohort, the association between job insecurity and decreased retirement incidence $(\mathrm{HR}=0.69,95 \% \mathrm{CI}$ $=0.50-0.95)$ was attenuated after adjustment for CHCs and covariates. In the U.S cohort, adjustment for CHCs and other social and health factors significantly decreased this association $(\mathrm{HR}=0.60,95 \% \mathrm{CI}=0.36-0.99)$, indicating that $\mathrm{CHCs}$, social, and health factors are contributing mechanistic factors underpinning retirement incidence in the U.S.

Conclusions The country level differences we observed may be driven by macro level factors operating latently, which may affect the work environment, health outcomes, and retirement decisions uniquely in different settings.

\section{Biomarkers}

\section{0-123 DNA DAMAGE IN LYMPHOCYTES OF FEMALE FARMERS MEASURED USING THE ALKALINE COMET ASSAY.}

${ }^{1}$ Poppy Evenden, Yannick Lecluse, Pierre Lebailly, Stephanie Perrier, Mathilde Boulanger Matthieu Meryet-Figuière, Anne-Sophie Lacauve, Elodie Niez, Hervé Perdry, Elisa BoutetRobinet, Séverine Tual, Stefano Bonassi, Raphaël Delépée. 'ANTICIPE INSERM U1086, France

10.1136/OEM-2021-EPI.6 
Introduction Many studies have shown a link between exposure to pesticides and a higher level of DNA damage. However, longitudinal studies are rare and little is known about long term DNA damage and cancer risk in exposed subjects.

Objectives i) Quantify DNA damage for the women included in a French agricultural cohort at two time points, ii) study the relationship between DNA damage and agricultural tasks, and iii) between DNA damage and cancer incidence.

Methods 320 female agricultural workers were enrolled from 1997 to 2000 and completed a face-to-face questionnaire. 245 gave a blood sample at enrolment (T0). Ten years later, 104 donated another sample (T10). Using the comet assay with an internal historical negative control, we quantified DNA damage in PBMC on 200 nuclei using a 4-category visual scoring system.

Results At enrolment, there were no differences in DNA damage for farm owners/co-owners $(n=135,55 \%, p=0.84)$, or participants in livestock tasks within our study population $(\mathrm{n}=159,65 \%, \mathrm{p}=0.23)$. Pesticide exposure through application of anti-parasites to livestock $(n=138,56 \%, p=0.25)$ and disinfection of milking equipment $(n=117,48 \%, p=0.78)$ did not influence DNA damage. However, damage tended to be higher for those implicated in milking $(n=109,44 \%$, $\mathrm{p}=0.18)$. Women who carried out administrative $(\mathrm{n}=180$, $73 \%)$ and domestic tasks $(n=213,87 \%)$ tended to have lower damage scores $(\mathrm{p}=0.08$ and $\mathrm{p}=0.07$, respectively), as well as women who undertook other tasks had significantly lower damage $(\mathrm{p}=0.04)$ than those not involved. From enrolment to end of 2017, 37 incident cancer cases were diagnosed, of which 21 were breast cancer cases. No association was found between DNA damage and cancer incidence $(\mathrm{p}=0.45)$.

Conclusion Occupational exposure in the agricultural field could be evaluated using the comet assay. Women's involvement to tasks not related to farm upkeep show a lower level of DNA damage than those not undertaking these tasks.

\section{0-198 ALTERED LEVELS OF IMMUNE MARKERS AMONG MALE ROTATING NIGHT SHIFT WORKERS IN SPAIN - THE HORMONIT STUDY}

'Barbara Harding, Ruth Aguilar, Gemma Moncunill, Carlota Dobaño, Gemma Castaño Vinyals, Ana Espinosa, Kyriaki Papantoniou, Manolis Kogevinas. 'Barcelona Institute for Global Health, Spain

\subsection{6/OEM-2021-EPI.7}

Introduction Few preliminary studies suggest that night shift work is associated with a desynchronization of rhythmic immune parameters, which may explain in part the increased risk for infection, cardiometabolic disorders, and cancer in shift workers.

Objectives To examine how night shift work alters cellular immune markers.

Methods This study included 46 male rotating shift workers from a car industry in Barcelona, Spain, sampled twice toward the end of a 3-week night shift (22:00-06:00 hrs) and a 3week day shift (06:00-14:00 hrs) rotation. We collected four blood samples per worker; before and after during each shift (night shift at 06:00 and 14:00, day shift at 22:00 and 6:00). We measured 30 cytokines, chemokines and growth factors using Luminex technology and examined within-person variations in analytes between sampling time-points and shifts. We applied linear mixed models to examine within-person associations between shift and analytes, comparing samples taken at 6:00am during each shift. We also conducted a factor analysis using analyte levels from all 4 time points for each individual to identify common factors and determine if these factors were altered by shift work.

Results We observed lower levels of several analytes during the night shift (cytokines IL17, IL12, IL4, TNF $\alpha$, and cytokine receptors IL1RA, IL2R; chemokines IP10, MIP1 $\alpha$, MIP1 $\beta$, and RANTES; growth factors GCSF, FGF, EGF, HGF, and VEGF) compared to the day shift. In the factor analysis, the main factor (explaining 57\% of the variance) was negatively associated with night shift (beta: $-0.14,95 \% \mathrm{CI}-0.25$ to -0.03 , pvalue less than 0.01), indicating that night shift affects immune marker levels in this pathway. This factor included growth factors (FGF, EGF, HGF), Th1 type cytokines (IL15, IL2) and pro-inflammatory cytokines and chemokines (IL1 $\beta$, MIP1 $\alpha$, MIP1 $\beta)$.

Conclusion Our results show that night shift is associated with disruption in multiple immune response pathways.

\section{0-242 LITERATURE REVIEW OF BIOLOGICAL MONITORING STUDIES TO ASSESS HEALTHCARE WORKER'S EXPOSURE TO ANTINEOPLASTIC DRUGS}

${ }^{1}$ Naqiyah Motiwala, Chun-Yip Hon. ${ }^{1}$ Ryerson University, Canada

\subsection{6/OEM-2021-EPI.8}

Introduction Antineoplastic drugs, also known as hazardous drugs, are used to treat cancer. Healthcare workers' exposure to these agents may lead to DNA damage and adverse reproductive effects. Exposure to antineoplastic drugs can be assessed via biological monitoring.

Objectives To conduct a literature review of studies examining biological monitoring of healthcare workers exposed to antineoplastic drugs to identify trends and knowledge gaps.

Methods The literature search was performed on three databases: Proquest, Web of Science and PubMed. For inclusion, an article had to be in English and published between 2005 and 2020. Search terms included 'antineoplastic drugs' or 'hazardous drugs' AND 'occupational' or 'healthcare workers' or 'pharmacist' or 'nurse' AND 'blood' or 'urine' or 'biomarkers'.

Results Overall, the literature review resulted in 70 unique articles - 30 examined urine as the matrix, 31 had non-urine samples and nine papers collected both urine and non-urine samples. Regarding the urine sampling studies, researchers collected either spot samples or 24-hr voids. Cyclophosphamide was the most common analyte (69\%) with a detection limit ranging from 0.09 to $0.20 \mathrm{ng} / \mathrm{ml}$. Approximately $75 \%$ of the articles reported at least one urine sample had detectable levels of drug. In some instances, urinary drug contamination was found even though workers wore personal protective equipment. For those studies that collected other matrices, blood and exfoliated buccal cells were the most common specimens. A majority (81\%) reported that there was a statistically significant difference in results between exposed and non-exposed populations. Many studies reported that safe work practices, which includes the use of personal protective equipment, may not be adequate to protect healthcare workers. 